\section{Possible Role of Birds in the Maintenance of Yellow Fever in West Africa}

IN 1936 it was shown that the blood of monkeys from endemic yellow fever zones in Africa contained immune bodies to the yellow fever virus, as judged by the intraperitoneal mouse protection test: the sera of monkeys outside the endemic zone did not contain immune bodies ${ }^{1}$. These results have since been confirmed by other workers. At the same time, the blood of a sheep from the Gambia was found to possess virucidal bodies against the yellow fever virus. Since then virucidal bodies have been found in the blood of cattle, sheep, dogs, pigs and a camel : the significance of these findings in domestic animals awaits further study, since virucidal bodies were present in the blood of certain domestic animals living outside the commonly accepted yellow fever zone.

Although there is highly suggestive evidence that monkeys may act as an alternative host to man in maintaining yellow fever in Africa, the monkey population is not large, nor is there evidence that monkeys migrate over great distances.

A search has therefore been made for other possible hosts sufficiently numerous to maintain the virus in the absence of man. The sera of a number of animals and birds have been examined from the Gold Coast. Although no epidemic has been reported in this Colony since 1937, single sporadic cases have occurred every year, and immunity surveys in children show that the number of cases reported bears no relation to the number of children giving positive results. Apart from monkey sera giving positive results, a rodent, a Cutting grass Thryonomys swinderianus, one of five examined, has been found to possess virucidal bodies to yellow fever virus in its blood. The sera of civet cats, giant rats, bush-tailed porcupines and royal pythons have been negative. More striking results have been obtained with the sera of wild birds. Of forty birds examined four have shown virucidal bodies to the yellow fever virus. The positive birds were a buff-backed heron Bubulcus ibis ibis, one of four examined, an African barn-owl Tyto alba affinis, one of three examined, and a Senegal kingfisher Halcyon senegalensis senegalensis, the only specimen examined. All these birds belong to common and widely distributed species.

Further work, which is at present proceeding, is required to determine the exact significance of the virucidal bodies contained in the sera of these birds. Should further evidence show that the virucidal action is due to the development of immune. bodies as a result of previous infection with the yellow fever virus, a clue may have been found to the maintenance of yellow fever in Africa, and possibly also an explanation of the occasional occurrence of sporadic infections in man at considerable intervals in space and time. Much further knowledge is required of the migration of birds within Africa. Thus the Senegal kingfisher is said to be migratory in Darfur, but there is no record of its migration in the western part of its range. The buff-backed heron, however, is known to have an extensive migratory range from the southern edge of the Sahara to the Guinea coast, thus traversing the endemic yellow fever zone from north to south. This bird is closely associated with man and with cattle.

It will be recalled that in America birds appear to play an important part in the maintenance of the eastern and western strains of equine encephalomyelitis virus : the same may be true of the yellow fever virus in Africa.

G. M. Findlay.

T. A. Cockburn.

${ }^{1}$ Findlay, G. M., Stefanopoulo, G. J., Davey, T. H., and Mahaffy, A. F., Trans. Roy. Soc. Trop. Med. and Hyg., 29, 419 (1936).

\section{Synergistic Effect of Para-aminobenzoic Acid and Sulphapyridine on Penicillin}

A solutron of sodium penicillin, containing 1,200 units per c.c., was tested for bacteriostatic activity against $B$. subtilis and found to be active in a dilution of $1: 100$ in a synthetic medium containing $0 \cdot 1$ per cent acid hydrolysed casein 1 .

When $p$-aminobenzoic acid was added to the medium in dilutions of $1: 2,500-1: 10,000$, the inhibitory effect of penicillin on the test organism was increased to a titre of $1: 6,000$. An increase in the activity of penicillin on addition of $p$-aminobenzoic acid was also observed with glucose broth as nutrient medium. A similar, though less marked, effect on penicillin activity was shown by $p$-aminobenzoic acid against Staphylococcus aureus (strain 663) in the same synthetic medium containing hydrolysed casein. Using a solution of sodium penicillin with a titre of $1: 40,000$, the effect of $p$-aminobenzoic acid (added in a dilution of $1: 2,500-1: 50,000$ ) was to increase the titre to $1: 75,000-1: 100,000$. $p$-Aminobenzoic acid had no effect on the activity of penicillin solution against Streptococcus hoemolyticus (strain 618, group A).

$p$-Aminobenzoic acid did not increase the activity of penicillin in concentrations greater than $1: 1,000$.

The enhancing effect of sulphapyridine on penicillin was more marked than that of $p$-aminobenzoic acid. Sulphapyridine, which, when tested against staphylococcus (strain 663), showed no inhibition of growth in digest broth in dilutions up to $1: 2,000$, was added in dilutions up to $1: 50,000$ to a solution of sodium penicillin, which inhibited at $1: 30,000$; inhibition occurred up to a titre of $1: 70,000$. When $p$-aminobenzoic acid was added in a dilution of $1: 20,000$ to the penicillin solution containing sulphapyridine (1:5,000), inhibition occurred at $1: 40,000$.

The experiment was repeated using 1 per cent. glucose broth, and the activity of penicillin against staphylococcus (strain 663) and streptococcus (strain 618) was again shown to be enhanced in presence of sulphapyridine. A solution of sodium penicillin inhibited staphylococcus at a titre of $1: 20,000$; in presence of sulphapyridine (in dilutions up to $1: 50,000)$ the solution inhibited at a titre of 1 : 50,000. Similarly, a solution of sodium penicillin inhibited streptococcus (strain 618 ) at dilutions up to $1: 50,000$; in presence of sulphapyridine (in dilutions from $1: 5,000$ to $1: 50,000)$, the same penicillin solution inhibited streptococcus at a dilution of 1 : 100,000 . Sulphapyridine itself showed inhibition only at a concentration of $\mathrm{J}: 2,500$. A dilution of $1: 5,000$ had no effect on the growth of steptococcus.

Thus, the inhibitory effect of penicillin in presence of small quantities of sulphapyridine, which, per se, are insufficient to show any effect on staphylococcus or streptococcus, is at least doubled.

Having shown in vitro the synergistic effect of penicillin and sulphapyridine, their action was investigated in vivo in mice, and some preliminary 